\title{
Transformation of Statistics in Fractional Quantum Hall Systems
}

\author{
$\underline{\text { John J. Quinn }}{ }^{1}$, Arkadiusz Wójs ${ }^{1,2}$, Jennifer J. Quinn ${ }^{3}$, and Arthur T. Benjamin ${ }^{4}$ \\ ${ }^{1}$ University of Tennessee, Knoxville, Tennessee 37996, USA \\ ${ }^{2}$ Wroclaw University of Technology, Wroclaw 50-370, Poland \\ ${ }^{3}$ Occidental College, Los Angeles, California 90041 \\ ${ }^{4}$ Harvey Mudd College, Claremont, California 91711
}

\begin{abstract}
A Fermion to Boson transformation is accomplished by attaching to each Fermion a tube carrying a single quantum of flux oriented opposite to the applied magnetic field. When the mean field approximation is made in Haldane's spherical geometry, the Fermion angular momentum $l_{F}$ is replaced by $l_{B}=l_{F}-\frac{1}{2}(N-1)$. The set of allowed total angular momentum multiplets is identical in the two different pictures. The Fermion and Boson energy spectra in the presence of many body interactions are identical only if the pseudopotential $V$ (interaction energy as a function of pair angular momentum $L_{12}$ ) increases as $L_{12}\left(L_{12}+1\right)$. Similar bands of low energy states occur in the two spectra if $V$ increases more quickly than this.
\end{abstract}

PACS: 71.10.Pm, 73.20.Dx, 73.40.Hm

Keywords: Fermion-Boson mapping; Fractional quantum Hall effect; Composite Fermion

Introduction. In two dimensional systems particle statistics can be changed by making a Chern-Simons (CS) transformation [1]. This transformation can be described as attaching to each particle an infinitesimal flux tube carrying a fictitious flux $\phi$ and a fictitious charge $q$, which couples to the vector potential produced by the flux tubes on every other particle in the standard way. If $q$ is equal to the electron charge and $\phi$ is an even number times $\phi_{0}=h c / e$, the quantum of flux, no change in statistics occurs. If $\phi$ is and odd number times $\phi_{0}$, Fermions are transformed into Bosons. The "gauge field" interactions associated with the fictitious charge and vector potential produced by the fictitious flux make the Hamiltonian of the system considerably more complicated. Only when the mean field approximation is made does the problem simplify. Jain [2] introduced the mean field CS transformation to give a simple intuitive picture of the hierarchy of fractional quantum Hall (FQH) states in terms of the resulting composite Fermions $(\mathrm{CF})$. Shortly after the introduction of the CF picture, Xie et al. [3] introduced the Fermion-Boson mapping connecting a 2D Fermion system at filling factor $\nu_{F}$ to a $2 \mathrm{D}$ Boson system with filling factor $\nu_{B}=\nu_{F}\left(1-\nu_{F}\right)^{-1}$. These authors noted that the size of the Hilbert space for the Fermion and Boson systems was identical, and they found that the $\mathrm{F} \rightarrow \mathrm{B}$ mapping accurately transformed the ground state of the Fermion system into that of the Boson system only if these ground states were incompressible FQH states. In this paper we show that the $\mathrm{F} \rightarrow \mathrm{B}$ transformation leads to identical energy spectra if and only if the pseudopotential $V\left(L_{12}\right)$ describing the interaction energy as a function of the pair angular momentum $L_{12}$ is of the "harmonic" form $V_{H}\left(L_{12}\right)=A+B L_{12}\left(L_{12}+1\right)$ where $A$ and $B$ are constants. Laughlin [4] correlations occur when the actual pseudopotential $V\left(L_{12}\right)$ rises more quickly with increasing $L_{12}$ than $V_{H}\left(L_{12}\right)$. Anharmonic effects (due to $\left.\Delta V\left(L_{12}\right)=V\left(L_{12}\right)-V_{H}\left(L_{12}\right)\right)$ cause the interacting Fermion and interacting Boson spectra to differ for every value of the filling factor.

It is well known that in the Haldane spherical geometry [5] the mean field CF transformation changes an electron of angular momentum $l_{F}$ to a $\mathrm{CF}$ of angular momentum $l_{F}^{*}=\left|l_{F}-p(N-1)\right|[6]$. Here $l_{F}=S_{F}$, one half the strength (measured in units of $\phi_{0}$ ) of 
the magnetic monopole which produces the radial magnetic field $B=2 S_{F} \phi_{0}\left(4 \pi R^{2}\right)^{-1}$ at the spherical surface of radius $R$ on which the $N$ electrons are confined, and $p$ is an integer. For an $N$ Boson system, the composite Boson transformation replaces $l_{B}$ by $l_{B}^{*}=\left|l_{B}-p(N-1)\right|$. In the $\mathrm{F} \rightarrow \mathrm{B}$ mapping $l_{F}$ is replaced by $l_{B}=\left|l_{F}-\frac{1}{2}(N-1)\right|$.

Angular Momentum Addition: Useful Theorems. When a shell of angular momentum $l$ contains $N$ identical particles (Fermions or Bosons), the resulting $N$ particle states can be classified by eigenvectors $|L, M, \alpha\rangle$, where $L$ is the total angular momentum, $M$ its $z$-component, and $\alpha$ a label which distinguishes independent multiplets with the same $L$. In the mean field CF $(\mathrm{CB})$ transformation $l_{F}\left(l_{B}\right)$ is transformed to $l_{F}^{*}\left(l_{B}^{*}\right)$. In trying to understand why the mean field $\mathrm{CF}$ picture correctly predicted the low lying band of states in the electron spectrum, several simple conjectures were proposed on the basis of numerical studies of a finite number of particles [7]. These conjectures have been elevated to the status of theorems by rigorous mathematical proof [8] using partition theory.

Theorem 1. The set of allowed total angular momentum multiplets of $N$ Fermions each with angular momentum $l_{F}^{*}$ is a subset of the set of allowed multiplets of $N$ Fermions each with angular momentum $l_{F}=l_{F}^{*}+(N-1)$.

Theorem 2. The set of allowed total angular momentum multiplets of $N$ Bosons each with angular momentum $l_{B}$ is identical to the set of multiplets for $N$ Fermions each with angular momentum $l_{F}=l_{B}+\frac{1}{2}(N-1)$.

From Theorem 2 it follows immediately that Theorem 1 also applies to Bosons. Theorem 2 is a stronger statement than a simple equality of sizes of the many body Hilbert spaces [3].

Interaction Effects. It has been shown that for the harmonic pseudopotential $V_{H}\left(L_{12}\right)$ the energy of any multiplet of angular momentum $L$ is given by $E_{\alpha}(L)=A \times \frac{1}{2} N(N-1)+$ $B \times\{N(N-2) l(l+1)+L(L+1)\}$. The energy is independent of $\alpha$, so that every multiplet with the same value of $L$ has the same energy. If $B_{F}=B_{B}=B$, then the spectrum of $N$ Bosons each with angular momentum $l_{B}$ is identical (up to a constant) to that of $N$ Fermions each with angular momentum $l_{F}=l_{B}+\frac{1}{2}(N-1)$. This is a rather surprising result because Fermions and Bosons sample different sets of values of the pair angular momentum. For example, for $N=9$ and $l_{F}=12$ (corresponding to $\nu_{F}=\frac{1}{3}$ ) the allowed values of the Fermion pair angular momentum consist of all odd integers between 1 and 23; for the corresponding Boson system with $l_{B}=8\left(\nu_{B}=\frac{1}{2}\right)$, the allowed values of $L_{12}$ are all even integers between 0 and 16 .

Xie et al. [3] determined the Boson and Fermion eigenfunctions by exact numerical diagonalization for six particle systems connected through the $\mathrm{F} \rightarrow \mathrm{B}$ transformation. They then transformed the Boson eigenfunctions into Fermion eigenfunctions by multiplying them by $\prod_{i<j}\left(z_{i}-z_{j}\right)$, as required by the $\mathrm{B} \rightarrow \mathrm{F}$ transformation. The overlap of these transformed Boson eigenfunctions with the exact Fermion eigenfunctions was then evaluated. The overlap was close to unity for incompressible quantum fluid states when the full Coulomb interaction was used, but it was considerably smaller when certain model pseudopotentials were used.

We have evaluated numerically the eigenstates of an eight electron system at $2 S_{F}=19$ to 23 (these states correspond to Laughlin $\nu_{F}=\frac{1}{3}$ states with zero, one, or two QP's) for a number of different pseudopotentials. In frames $\left(a-a^{\prime \prime}\right)$ and $\left(b-b^{\prime \prime}\right)$ of Fig. 1 1 we contrast the energy spectra for the Fermion and Boson systems at $\nu_{F}=\frac{1}{3}\left(\nu_{B}=\frac{1}{2}\right)$ for the Coulomb pseudopotential appropriate for the lowest Landau level and for the model pseudopotentials 

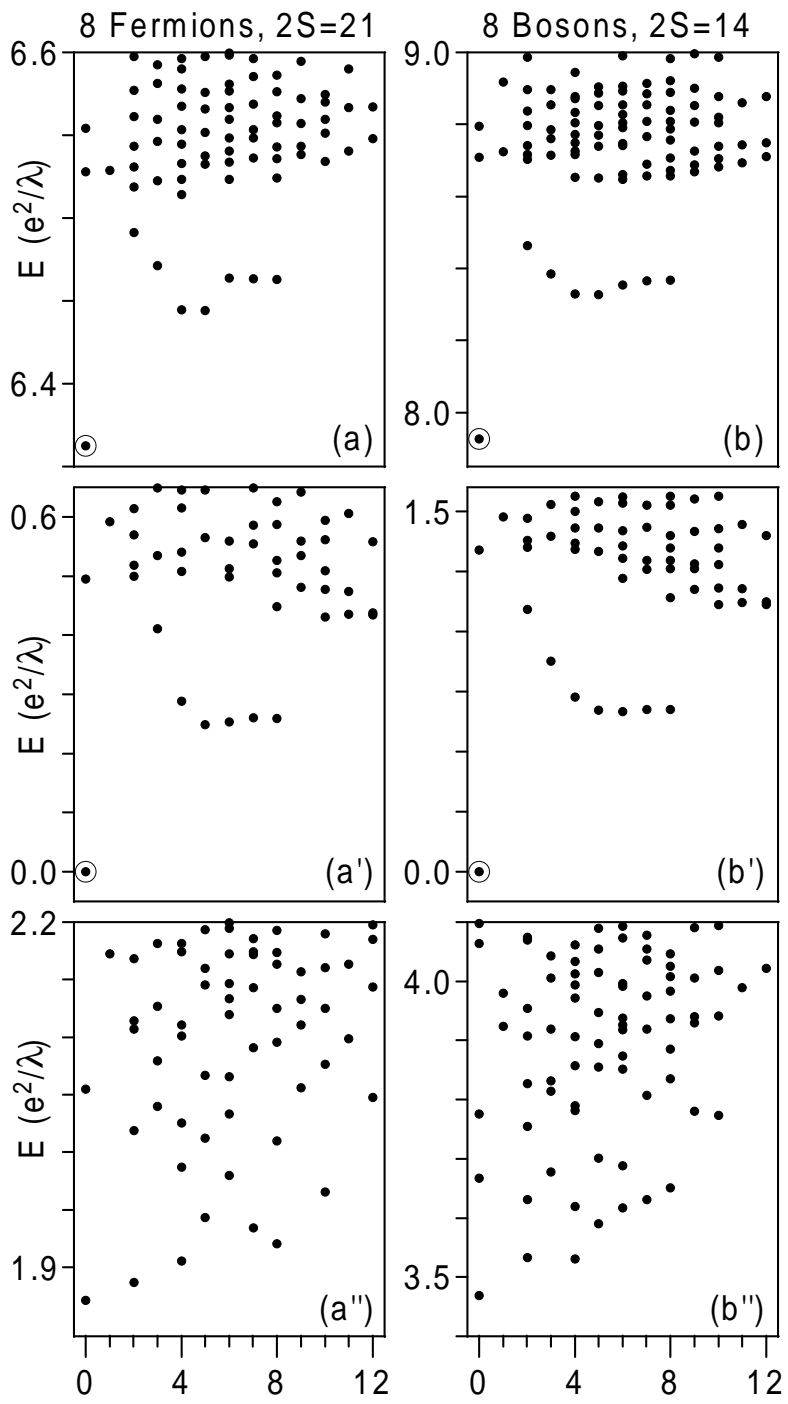

L

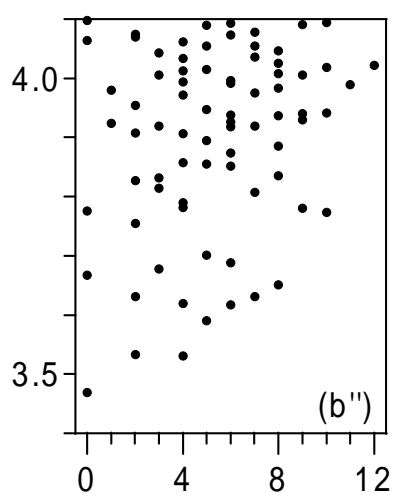

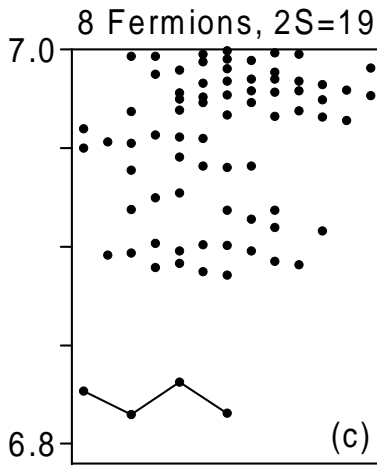
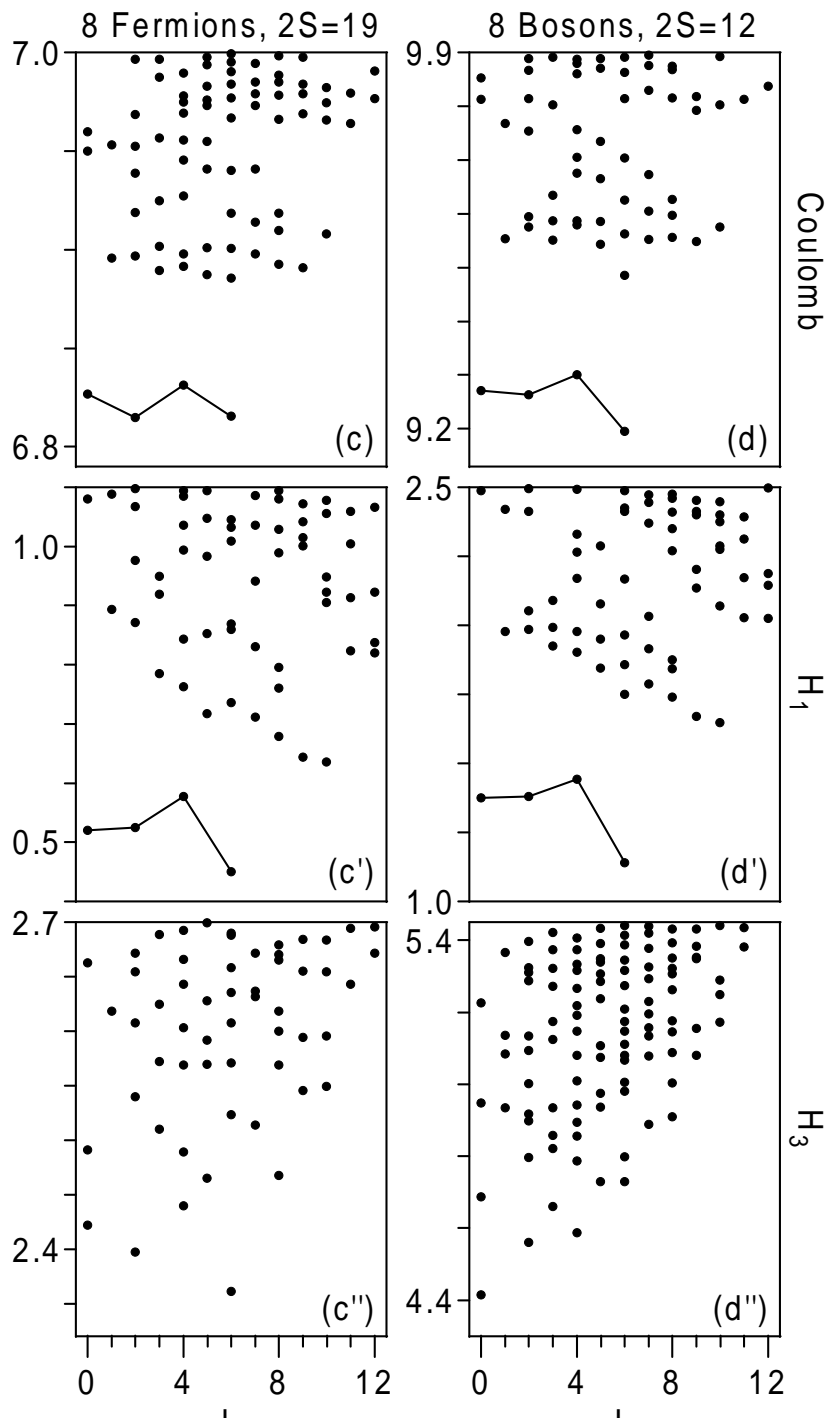

FIG. 1. The energy spectra (energy $E$ vs. angular momentum $L$ ) of eight Fermions at the monopole strength $2 S_{F}=21$ (filling factor $\nu_{F}=\frac{1}{3}$; circles) and 19 (two Laughlin quasielectrons; lines), and of eight Bosons at $2 S_{B}=14\left(\nu_{B}=\frac{1}{2}\right.$; circles) and 12 (two quasielectrons; lines) for the Coulomb pseudopotential in the lowest Landau level (a-d), and for the model pseudopotentials $H_{1}$ $\left(\mathrm{a}^{\prime}-\mathrm{d}^{\prime}\right)$, and $H_{3}\left(\mathrm{a}^{\prime \prime}-\mathrm{d}^{\prime \prime}\right) . \lambda$ is the magnetic length.

$H_{1}$ and $H_{3} . \quad H_{1}$ is defined to have only the largest pseudopotential coefficient $\left[V\left(L_{12}=\right.\right.$ $\left.2 l_{F}-1\right)$ for Fermions and $V\left(L_{12}=2 l_{B}\right)$ for Bosons] equal to its Coulomb value and all other pseudopotential coefficients equal to zero. $H_{3}$ has the two largest pseudopotential coefficients equal to their Coulomb values and all other coefficients equal to zero. In frames $\left(\mathrm{c}-\mathrm{c}^{\prime \prime}\right)$ and $\left(\mathrm{d}-\mathrm{d}^{\prime \prime}\right)$ we do the same for the state containing two Laughlin quasielectrons (QE). The lowest states in $\left(\mathrm{a}, \mathrm{a}^{\prime}, \mathrm{b}, \mathrm{b}^{\prime}\right)$ are quite similar consisting of a Laughlin $L=0$ ground state. The magnetoroton band (at $2 \leq L \leq 8$ ) is apparent in all four spectra, although the gaps and band widths are different for different pseudopotentials. The lowest states in (c, $\left.\mathrm{c}^{\prime}, \mathrm{d}, \mathrm{d}^{\prime}\right)$ are also similar containing two QE's with $l_{\mathrm{QE}}=\frac{1}{2}(N-1)=\frac{7}{2}$ giving $L=N-2$, 
$N-4, \ldots=0,2,4$, and 6 . The pseudopotential $H_{3}$ used in $\left(\mathrm{a}^{\prime \prime}-\mathrm{d}^{\prime \prime}\right)$ gives very different results. We believe that this behavior results because the pseudopotential used in $H_{3}$ is not "short range" in the sense that it does not increase faster than $V_{H}\left(L_{12}\right)$ ) in the region of non-vanishing $V\left(L_{12}\right)$. This behavior of the pseudopotential results in correlations that are very different from Laughlin correlations, and it accounts for the poor overlap found by Xie et al. [3] for certain model pseudopotentials.

Quasiparticles. The $\mathrm{F} \rightarrow \mathrm{B}$ transformation allows us to better understand the Boson [5] vs. Fermion [9, 10] description of QP's in incompressible FQH states. Laughlin condensed states having $\nu_{F}=(2 p+1)^{-1}$ (where $p$ is a positive integer) occur at $2 S_{F}=(2 p+1)(N-$ 1) in the Haldane spherical geometry. The $\mathrm{CF}$ transformation gives an effective angular momentum $l_{F}^{*}=S_{F}^{*}=S-p(N-1)=\frac{1}{2}(N-1)$ when $2 p$ flux quanta are attached to each electron. Thus the $N$ CF's fill the $2 l^{*}+1$ states of the lowest CF shell giving an $L=0$ incompressible ground state.

The $\mathrm{F} \rightarrow \mathrm{B}$ transformation gives $2 S_{B}=2 S_{F}-(N-1)=2 p(N-1)$ and a Boson filling factor of $\nu_{B}=(2 p)^{-1}$. Making a CB transformation gives $l_{B}^{*}=S_{B}^{*}=S_{B}-p(N-1)=0$. This also gives an $L=0$ incompressible ground state because each CB has $l_{B}^{*}=0$. Thus the $\mathrm{CF}$ description of a Laughlin state has one filled CF shell of angular momentum $l_{F}^{*}=\frac{1}{2}(N-1)$, while the CB description has $N$ CB's each with angular momentum $l_{B}^{*}=0$.

For $2 S_{B}=2 p(N-1) \pm n_{\mathrm{QP}}$, where the + and - occur for quasiholes $(\mathrm{QH})$ and quasielectrons $(\mathrm{QE})$, respectively, we define $2 l_{B}^{*}=\left|2 S_{B}^{*}\right|=n_{\mathrm{QP}}$. This gives exactly the same set of angular momentum multiplets as obtained in the CF picture with $2 S_{F}=(2 p+1)(N-1) \pm n_{\mathrm{QP}}$ only if a hard core repulsion forbids the Boson QE pair from having the largest allowed pair angular momentum $L_{12}^{\mathrm{MAX}}=N$ [11]. This behavior is observed in Fig. 1 $1\left(\mathrm{c}-\mathrm{c}^{\prime}, \mathrm{d}^{-} \mathrm{d}^{\prime}\right)$ where the Boson treatment of two QE's predicts states at $L=0,2,4,6$, and 8 , but the $L=8$ state does not occur in the low energy band. Since the description of CB's (with hard core QE interaction) and CF's give identical sets of QP states, filled QP levels (implying daughter states) occur at identical values of the applied magnetic field.

Acknowledgment. John J. Quinn acknowledges partial support from the Materials Research Program of Basic Energy Sciences, US Department of Energy.

[1] See, for example, F. Wilczek, Fractional Statistics and Anyon Superconductivity (World Scientific, Singapore, 1990) and references therein.

[2] J. Jain, Phys. Rev. Lett. 63, 199 (1989).

[3] X. C. Xie, S. He, and S. Das Sarma, Phys. Rev. Lett. 66, 389 (1991).

[4] R. Laughlin, Phys. Rev. Lett. 50, 1395 (1983).

[5] F. D. M. Haldane, Phys. Rev. Lett. 51, 605 (1983).

[6] X. M. Chen and J. J. Quinn, Solid State Commun. 92, 865 (1994).

[7] A. Wójs and J. J. Quinn, Solid State Commun. 108, 493 (1998); ibid 110, 45 (1999); Acta Phys. Pol. A 96, 593 (1999); Phil. Mag. B 80, 1405 (2000).

[8] J. J. Quinn, A. Wójs, J. J. Quinn, and A. T. Benjamin, unpublished (cond-mat/0007481).

[9] P. Sitko, K.-S. Yi, and J. J. Quinn, Phys. Rev. B 56, 12417 (1997).

[10] A. Wójs, and J. J. Quinn, Phys. Rev. B 61, 2846 (2000).

[11] S. He, X.-C. Xie, and F.-C. Zhang, Phys. Rev. Lett. 68, 3460 (1992). 